\title{
Multiepoch Spectropolarimetry of SN 2011fe
}

\author{
Peter A. Milne ${ }^{1}$, G. Grant Williams ${ }^{1,2}$, Amber Porter ${ }^{3}$, Paul S. Smith ${ }^{1}$, Nathan Smith ${ }^{1}$, Mark D. Leising ${ }^{3}$, \\ Buell T. Jannuzi ${ }^{1}$, and E. M. Green ${ }^{1}$ \\ ${ }^{1}$ University of Arizona, Steward Observatory, 933 N. Cherry Avenue, Tucson, AZ 85721, USA \\ ${ }^{2}$ MMT Observatory, 933 N. Cherry Avenue, Tucson, AZ 85721, USA \\ 3118 Kinard Laboratory, Clemson University, Clemson, SC 29634, USA \\ Received 2016 June 7; revised 2016 November 18; accepted 2016 November 21; published 2017 January 20
}

\begin{abstract}
We present multiple spectropolarimetric observations of the nearby Type Ia supernova (SN) 2011fe in M101, obtained before, during, and after the time of maximum apparent visual brightness. The excellent time coverage of our spectropolarimetry has allowed better monitoring of the evolution of polarization features than is typical, which has allowed us new insight into the nature of normal SNe Ia. SN $2011 \mathrm{fe}$ exhibits time-dependent polarization in both the continuum and strong absorption lines. At early epochs, red wavelengths exhibit a degree of continuum polarization of up to $0.4 \%$, likely indicative of a mild asymmetry in the electron-scattering photosphere. This behavior is more common in subluminous SNe Ia than in normal events, such as SN 2011fe. The degree of polarization across a collection of absorption lines varies dramatically from epoch to epoch. During the earliest epoch, a $\lambda 4600-5000 \AA$ complex of absorption lines shows enhanced polarization at a different position angle than the continuum. We explore the origin of these features, presenting a few possible interpretations, without arriving at a single favored ion. During two epochs near maximum, the dominant polarization feature is associated with the Si II $\lambda 6355 \AA$ absorption line. This is common for SNe Ia, but for SN $2011 \mathrm{fe}$ the polarization of this feature increases after maximum light, whereas for other SNe Ia, that polarization feature was strongest before maximum light.
\end{abstract}

Key words: supernovae: general

\section{Introduction}

Type Ia supernova (SN Ia) explosions convey information about the nucleosynthesis by the thermonuclear destruction of a CO white dwarf (Iwamoto et al. 1999), and they provide a way to measure the expansion of the universe by using their peak magnitudes as standardizable candles (Phillips 1993). The unexpected finding that the expansion of the universe is accelerating (Riess et al. 1998; Perlmutter et al. 1999) has focused interest on a better understanding of the SN Ia explosion mechanism. It has long been recognized that there are variations within the SN Ia category. More luminous events rise to a peak and decline from a peak on a longer timescale than less luminous events (Branch et al. 1993; Phillips 1993). The majority of events fall within a "normal" grouping, although some cases have been recognized where the luminosity does not correlate with peak width (e.g., Benetti et al. 2005; Wang et al. 2009; Foley \& Kasen 2011). Asymmetries in the explosion may hold important clues to the explosion mechanism itself, as well as to the consequent diversity in observed properties.

Spectropolarimetry has emerged as a powerful probe of SNe Ia (see Livio \& Pringle 2011) and of the intervening interstellar (ISM) or circumstellar (CSM) material (Patat et al. 2015; Porter et al. 2016). The degree of polarization of the continuum emission is generally lower for SNe Ia than for core-collapse events, but it has been detected at significant levels for a range of SN Ia subclasses (Wang \& Wheeler 2008). Polarization at the wavelengths of observed absorption lines is particularly interesting as it affords the opportunity to study the distribution of specific elements within the ejecta. This "line polarization" has been observed to change markedly near maximum light. The signature Si II $\lambda 6355 \AA$ line in addition to the Ca II near-infrared (NIR) triplet near $8000 \AA$ has exhibited polarization in a number of SNe Ia, including 2001el (Wang et al. 2003), 2002bo (Wang et al. 2007), 1997dt, 2002bf, 2003du (Leonard et al. 2005), 2004S (Chornock \& Filippenko 2008), 2004dt (Leonard et al. 2005; Wang et al. 2006), 2006X (Patat et al. 2009), 2008fp (Cox \& Patat 2014), 2012fr (Maund et al. 2013), and 2014J (Patat et al. 2015). Likewise, the subluminous 1999by (Howell et al. 2001), 2005ke (Patat et al. 2012), and the super-Chandrasekhar explosion 2009dc (Tanaka et al. 2010) have shown variable polarization in these lines. Other elements have been identified in polarization spectra, notably Fe II lines in SNe 1997dt (Leonard et al. 2005) and 2004S (Chornock \& Filippenko 2008), and Mg II in SN 2004dt (Wang et al. 2006) and SN 2006X (Patat et al. 2009). For a review of polarimetric studies of SNe Ia, see Wang \& Wheeler (2008).

SN 2011fe occurred in M101 and was discovered on 2011 August 24 by the Palomar Transient Factory (PTF; Nugent et al. 2011a). The proximity of M101, $\sim 6.2 \mathrm{Mpc}$, and indications that the SN suffered minimal host-galaxy extinction suggested that the SN would become the brightest SN Ia since SN 1972E. Studies of the light curve suggest that the SN was discovered just 0.5 day after the explosion, and the explosion time is constrained to very high precision (Nugent et al. 2011b). Optical spectra revealed SN 2011fe to be a normal SN Ia, with detections of C II $\lambda 6580 \AA$ and $\lambda 7234 \AA$ in absorption (Cenko et al. 2011; Parrent et al. 2012; Pereira et al. 2013), and the blueshift of Si II $\lambda 6355 \AA$ placed it in the low-velocity (LVG/LV) class (see Benetti et al. 2005; Wang et al. 2009; Foley \& Kasen 2011 for LVG/HVG and LV/HV definitions). Studies of preexplosion images of the site of SN 2011fe place the strictest upper limits yet on the luminosity of any SN Ia progenitor, arguing against a single-degenerate progenitor containing a giant donor star (Li et al. 2011). 
Table 1

SPOL Observation Log for SN $2011 \mathrm{fe}$

\begin{tabular}{|c|c|c|c|c|c|c|}
\hline UT Date & $\begin{array}{c}\text { Age } \\
+\exp ^{\mathrm{a}} \\
\text { (days) }\end{array}$ & $\begin{array}{c}\text { Age } \\
+B_{\max } \mathrm{b} \\
\text { (days) }\end{array}$ & $\begin{array}{c}\text { UT } \\
\text { (start) }\end{array}$ & Telescope ${ }^{c}$ & $\begin{array}{l}\text { Exp } \\
\text { (s) }\end{array}$ & Epoch \\
\hline 2011 Aug 29 & 5.4 & -12.0 & $03: 32: 02$ & Bok & 3840 & 1 \\
\hline 2011 Sep 04 & 11.4 & -6.0 & $02: 51: 44$ & Kuiper & 4320 & 2 \\
\hline 2011 Sep 15 & 22 & 5 & $02: 53: 41$ & Bok & 1504 & 3 \\
\hline 2011 Sep 16 & 23 & 6 & 02:48:08 & Bok & 2784 & 3 \\
\hline 2011 Sep 26 & 33 & 16 & $02: 41: 28$ & Bok & 1440 & 4 \\
\hline 2011 Sep 28 & 35 & 18 & $02: 53: 44$ & Bok & 800 & 4 \\
\hline 2011 Sep 29 & 36 & 19 & $02: 20: 22$ & Bok & 1920 & 4 \\
\hline 2011 Sep 30 & 37 & 20 & 02:26:08 & Bok & 1920 & 4 \\
\hline 2011 Oct 01 & 38 & 21 & $02: 24: 33$ & Bok & 1280 & 4 \\
\hline 2011 Oct 06 & 43 & 26 & 02:09:04 & Bok & 1280 & 4 \\
\hline 2011 Nov 28 & 96 & 79 & $12: 38: 26$ & Kuiper & 320 & 5 \\
\hline 2011 Dec 27 & 125 & 108 & $11: 57: 12$ & Kuiper & 720 & 6 \\
\hline 2011 Dec 28 & 126 & 109 & $12: 32: 48$ & Kuiper & 480 & 6 \\
\hline 2011 Dec 29 & 127 & 110 & 12:08:05 & Kuiper & 720 & 6 \\
\hline 2011 Dec 30 & 128 & 111 & $11: 18: 47$ & Kuiper & 720 & 6 \\
\hline 2012 Jan 01 & 129 & 112 & $11: 33: 31$ & Kuiper & 720 & 6 \\
\hline 2012 Jan 26 & 155 & 138 & $11: 26: 55$ & Bok & 960 & 7 \\
\hline 2012 Jan 28 & 156 & 139 & 13:04:04 & Bok & 360 & 7 \\
\hline 2012 Feb 15 & 174 & 157 & $12: 47: 13$ & Bok & 360 & 8 \\
\hline 2012 Feb 21 & 180 & 163 & $12: 34: 54$ & Bok & 360 & 8 \\
\hline 2012 Mar 25 & 213 & 196 & $11: 41: 57$ & Bok & 480 & 9 \\
\hline 2012 Apr 16 & 235 & 218 & $10: 28: 33$ & MMT & 960 & 10 \\
\hline
\end{tabular}

Notes.

a Epoch relative to explosion, 2011 August 23.7, as estimated by averaging explosion dates of Brown et al. (2012b) and Nugent et al. (2011a).

${ }^{\mathrm{b}}$ Epoch relative to the reported date of $B_{\max }, 10.1 \pm 2011$ September 0.2, as reported by Matheson et al. (2012).

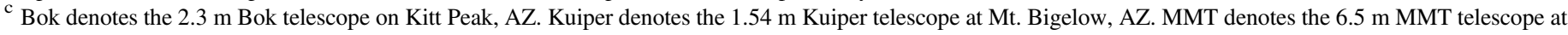
Mt. Hopkins, AZ. The same SPOL instrument was used for all observations.

Observations of the SN in the optical (Nugent et al. 2011b; Bloom et al. 2012) and ultraviolet (UV) and X-ray wavelength ranges (Brown et al. 2012a) constrained the exploding star to be smaller than main-sequence stars and further constrained the donor stars. The UV-optical colors demonstrated that the $\mathrm{SN}$ is of the NUV-blue subset of normal SNe Ia (Milne et al. 2013). Patat et al. (2013) reported a small change in a component of the Na I D absorption for SN 2011fe between +3 days and +19 days, a change that has been attributed to the presence of circumstellar material (Patat et al. 2007; Simon et al. 2009). However, by investigating reasonable expectations of the change of this absorption line with the geometrical increase in the emitting region compared to the size scale of variations in the ISM, Patet et al. (2013) found that the variation could be due to the ISM and not the CSM.

SN 2011fe was the nearest Type Ia explosion in several decades, providing an unprecedented opportunity to obtain spectropolarimetry of a normal LV SN Ia with modest-aperture telescopes. We initiated a campaign to obtain multiepoch spectropolarimetry of SN $2011 \mathrm{fe}$ at Steward Observatory using the $1.5 \mathrm{~m}$ Kuiper, the $2.3 \mathrm{~m} \mathrm{Bok}$, and the $6.5 \mathrm{~m} \mathrm{MMT}$ telescopes. We describe the results of these observations below.

\section{Observations}

The CCD Imaging/Spectropolarimeter (SPOL; Schmidt et al. 1992b) mounted on the Steward Observatory $2.3 \mathrm{~m}$ Bok telescope (Kitt Peak, AZ), the $1.54 \mathrm{~m}$ Kuiper telescope (Mt. Bigelow, AZ), and the $6.5 \mathrm{~m}$ MMT telescope (Mt. Hopkins, AZ) was used to obtain spectropolarimetry of SN 2011fe on 22 nights over 8 months. We have grouped those nights into 10 epochs in Table 1. The grouping was justified by a confirmed lack of internight variations in the $q$ or $u$ spectra during each epoch. Observations at the Kuiper and Bok telescopes used the 600 line $\mathrm{mm}^{-1}$ grating in first order with a 5"! $1 \times 51^{\prime \prime}$ slit and a Hoya L38 blocking filter providing a spectral resolution of 20 A from 4000-7550 $\AA$. Observations at the MMT used the 964 line $\mathrm{mm}^{-1}$ grating in first order with a 1 .'9 $919^{\prime \prime}$ slit and a Hoya L38 blocking filter providing a spectral resolution of $\sim 20 \AA$ from $4100-7200 \AA$. A rotatable semiachromatic halfwave plate was used to modulate incident polarization, and a Wollaston prism in the collimated beam separated the orthogonally polarized spectra onto a thinned, anti-reflectioncoated $800 \times 1200$ SITe CCD. The efficiency of the wave plate as a function of wavelength is measured by inserting a fully polarizing Nicol prism into the beam above the slit. A series of four separate exposures that sample 16 orientations of the wave plate yield two independent, background-subtracted measures of each of the normalized linear Stokes parameters, $q$ and $u$. Each night, several such sequences of observations of SN 2011fe were obtained and combined, with the weighting of the individual measurements based on photon statistics.

We confirmed that the instrumental polarization of SPOL mounted on the Bok, Kuiper, and MMT telescopes is much less than $0.1 \%$ through observations of the unpolarized standard stars BD+28 4211, HD 212311 and G191B2B (Schmidt et al. 1992a). The adopted correction from the instrumental to the standard equatorial frame for the linear polarized position angle on the sky $(\theta)$ for all epochs was determined from the average position angle offset of Hiltner 960 and VI Cyg \#12. Additional observations of the polarization standard stars $\mathrm{BD}+59^{\circ}$ 
389 and $\mathrm{BD}+64^{\circ} 106$ were made during the third epoch and of HD 245310 during the last epoch. Differences between the measured and expected polarization position angles were $<0.5$ for all of the standard stars.

During Epoch 1, two field stars within $\sim 2^{\prime}$ of SN $2011 \mathrm{fe}$ (2MASS J14031367+5415431 and 2MASS J14025413 +5416288 ) were measured to check for significant Galactic interstellar polarization (ISP) along the line of sight to the SN. These stars yielded a consistent estimate for Galactic ISP, with $P_{\max }=0.11 \pm 0.03 \%$ at $\theta=114^{\circ} \pm 7^{\circ}$ for 2MASS $\mathrm{J} 14031367+5415431$ and $P_{\max }=0.16 \pm 0.03 \%$ at $\theta=$ $109^{\circ} \pm 6^{\circ}$ for 2 MASS $\mathrm{J} 14025413+5416288$, assuming that $\lambda_{\max }$, the wavelength where the ISP is at a maximum $\left(P_{\max }\right)$, is $5550 \AA$ (Serkowski et al. 1975). The results for the field stars were averaged, and $P_{\max }=0.13 \%$ at $\theta=112^{\circ}$ was adopted as the Galactic ISP in the sight line to SN 2011fe. This low value for the Galactic ISP is consistent with the high Galactic latitude of M101 and the very low estimated amount of extinction, $E(B-V) \leqslant 0.1 \mathrm{mag}$, for the supernova (Foley \& Kirshner 2013). We reduced the data using custom but mature IRAF routines. We began by bias-subtracting and flat-fielding each image, and we used observations of $\mathrm{He}, \mathrm{Ne}$, and Ar lamps at the beginning of each run for wavelength calibration purposes. We then extracted Stokes paramters $q$ and $u$. We debiased the positive definite nature of the polarization calculation using the prescription $P=$ $\pm \sqrt{\left|Q^{2}+U^{2}-\frac{1}{2}\left(\sigma_{Q}^{2}+\sigma_{U}^{2}\right)\right|}$ (Wardle \& Kronberg 1974), where the sign is determined according to the sign of the modulus. Finally, to further increase the signal-to-noise ratio, we binned the Stokes parameters, to $16 \AA$ wide intervals.

Since our standard instrumental setup for the SPOL instrument does not reach to long enough wavelengths, this work does not include any observations of the Ca IR lines, which are strongly polarized in some SNe Ia. The standard setup is chosen to avoid the significant fringing present at wavelengths longer than $7000 \AA$.

\section{Polarization of SN 2011fe: Peak Epochs 1-4}

Our sequence of the first four epochs of spectra is shown in the top panel of Figure 1, displaying the emergence of absorption features typical of SNe Ia. The lower panels show the $q$ and $u$ polarization spectra, uncorrected for either Galactic polarization or host-galaxy ISP. Epochs 1-3 show clear signatures of line polarization in both the $q$ and $u$ spectra, while Epoch 4 appears to show no line polarization features. By Epoch 4 the spectrum is approaching the nebular phase, a time when the optical depth to electron scattering is low and thus the intrinsic SN polarization is expected to be low. Polarization observed in the nebular epoch is often assumed to be due to ISP. However, Figure 2 shows that some level of intrinsic line polarization remains at Epoch 4, leading us to employ a different method in this work, computing the inverse varianceweighted average of $q$ and $u$ for the line-free continuum region spanning $4600-5400 \AA$ for Epochs $4-10$. We find $q$ (ISP) $=-0.12 \pm 0.06 \%$ and $u$ (ISP) $=0.10 \pm 0.07 \%$. The error bars were determined by calculating the standard deviations of the individual epoch averages compared to $q$ (ISP) and $u$ (ISP). These values are shown as blue lines and the uncertainties as blue shading in Figure 2. For comparison, also shown in Figure 2 are the same values calculated across the entire 4000-7000 $\AA$ wavelength range (red lines and pink shading); the values are similar within the error bars. These

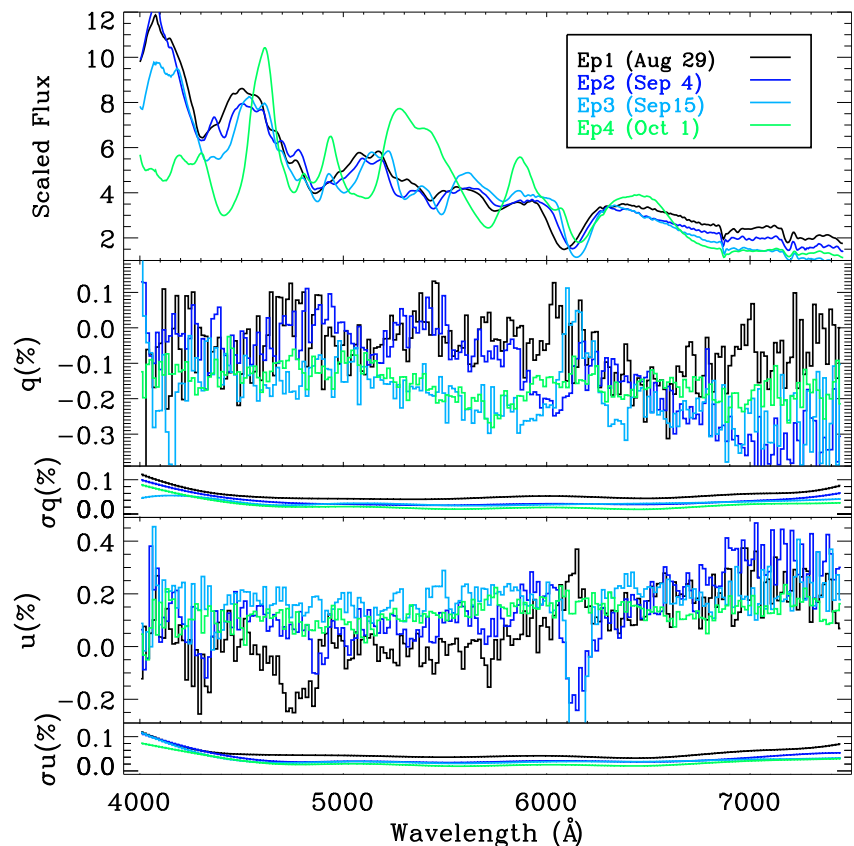

Figure 1. The first four epochs (color coded) of flux, and uncorrected Stokes parameters $q$ and $u$. The flux values have been scaled to the first epoch flux at $5800 \AA$ using the following flux ratios: 1.0, 0.228, 0.148, and 0.215 . The $q$ and $u$ spectra have been binned to $16 \AA$ bins, with the corresponding errors shown below each spectrum.

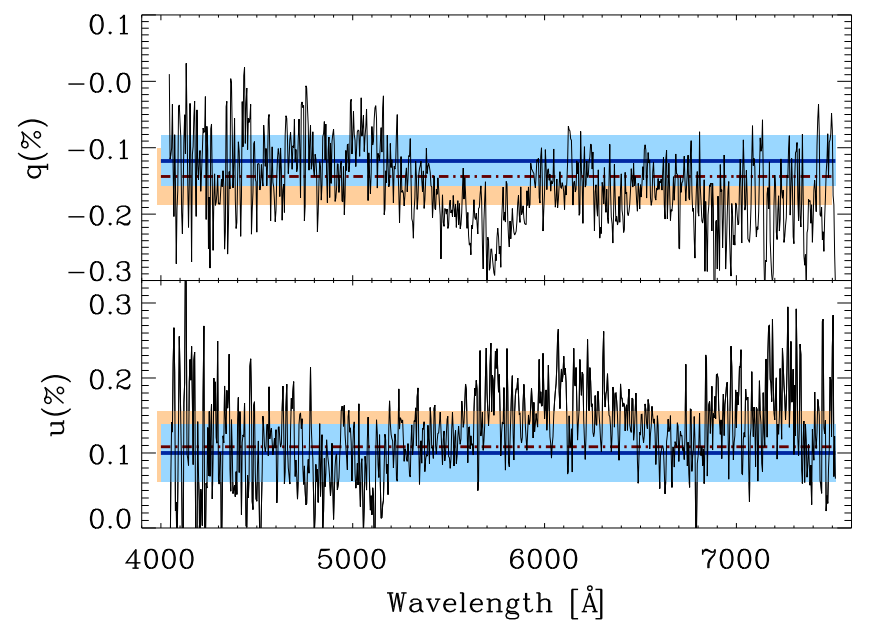

Figure 2. The $q$ and $u$ spectra of SN 2011fe from Epoch 4 compared to the values chosen for $q$ (ISP) and $u$ (ISP). The solid blue line shows the weighted average of the 4600-5400 ̊ wavelength range for Epochs 4-10. The dot-dashed red line shows the average from the wider 4000-7000 wavelength range for Epochs 4-10. The blue and red shaded regions show the standard deviations of the individual epoch averages compared to the overall average values.

error bars will be used in determining the uncertainties for the $P_{\text {Si II }}$ evolution (Section 4).

By using constants, we do not account for any possible wavelength dependence of the ISP, which we consider a minor effect because of the very small polarization levels. A small wavelength dependence would lead to our single ISP value being biased toward the value at $5000 \AA$. Figure 3 shows the flux, polarization, position angle, and $q$ and $u$ spectra after subtracting our determination of the total ISP, which includes both a Galactic and host-galaxy contribution. We did not attempt to separate Galactic and host ISP in this work, but we 


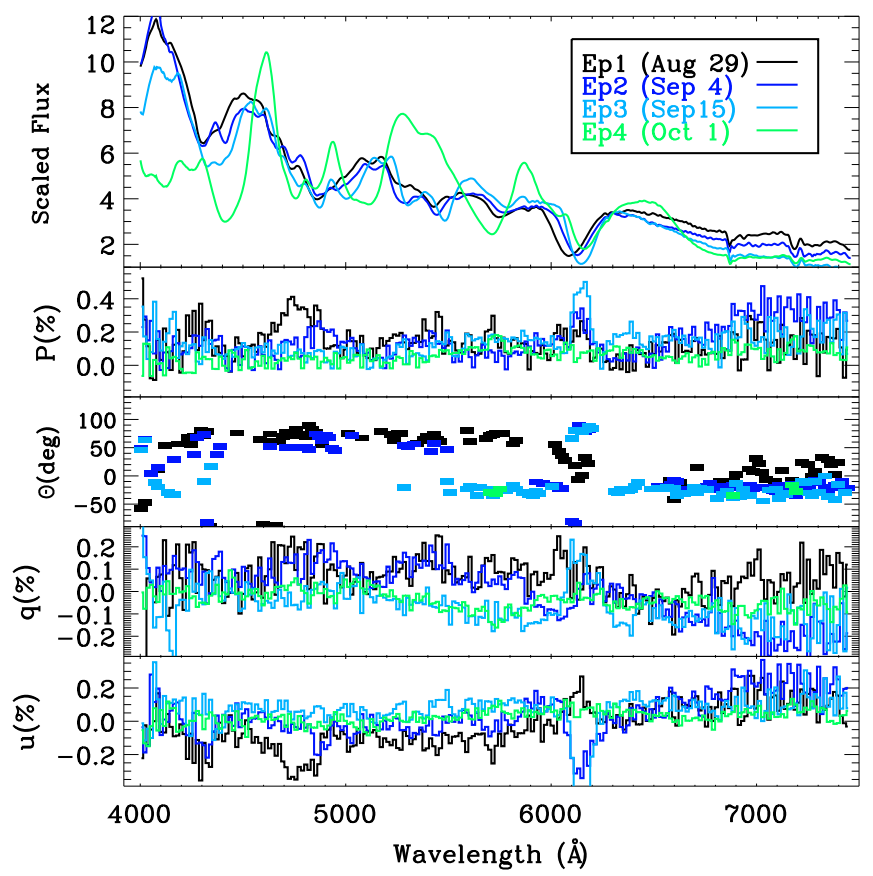

Figure 3. The first four epochs (color coded) of flux, degree of polarization, angle, and ISP-corrected Stokes parameters $q$ and $u$. The flux values have been scaled to the first epoch flux at $5800 \AA$ using the following flux ratios: 1.0 , $0.228,0.148$, and 0.215 . Here, $P$ and $\theta$ were determined with the ISP-corrected $q$ and $u$. For presentation purposes, $\theta$ was only plotted when $P>0.08 \%$. The $P, \theta, q$, and $u$ spectra have been binned into $16 \AA$ bins.

note that our estimates of the Galactic ISP, shown in Section 2, are close to the total ISP we derive above. For presentation purposes, $\theta$ is only plotted when $P>0.08 \%$.

\subsection{Spectral Fitting with SYNAPPS}

In order to study the nature of the line polarization, we employed the SYNAPPS algorithm (Thomas et al. 2011), which was derived from the SYNOW algorithm (Branch et al. 2005) to fit the three spectra that showed line polarization features. The input parameters of the SYNAPPS fits are shown in Table 2. We will discuss the SYNAPPS fitting before attempting to match line features with polarization features.

The upper panels of Figures 4-6 show the SYNAPPS fit to the Epoch 1-3 flux spectra, providing acceptable fits to these spectra, particularly in the region of line polarization features. The middle panels show the polarization $(P)$ and position angle $(\theta)$ and will be discussed in the next section. The lower panels of Figures 4-6 show the contributions of individual ions to the composite spectra, with the flux scaling factor listed along the right-hand axes to provide a sense of line strength. Different scaling factors were used to permit a better sense of the wavelength range for weaker features, with the scaling factor defined as the range compared to that of the Si II range.

The Epoch 1 spectrum has strong Si II (with both a photospheric and weaker high-velocity component) and Si III and $\mathrm{Mg}$ II absorption features. Iron and sulfur lines provide a broad wavelength range of weaker absorption. Also detected is $\mathrm{C}$ II, which is present in about one-third of SNe Ia and is interpreted to be a signature of unburned carbon (Parrent et al. 2011; Thomas et al. 2011; Folatelli et al. 2012; Silverman et al. 2012). The Epoch 2 fitting is similar to Epoch 1, but with the high-velocity (HV) Si II feature much weaker. Epoch 3 presents fewer lines, as the HV components of Si II and Fe II are no longer required, nor is $\mathrm{C} \mathrm{II}$ required, but now $\mathrm{Na} \mathrm{I}$ is required. Across the epochs, the blueshift of the photospheric Si II feature decreases gradually with epoch, consistent with an LV determination (Parrent et al. 2012). The multifeature Fe II, Fe III, and S II complexes persist across the epochs. These SYNAPPS fits can be compared to similar fits presented by Parrent et al. (2012) based upon optical spectra obtained as part of the PTF program.

\subsection{Line Polarization}

The middle panels of Figures 4-6 show the polarization $(P)$ and position angle $(\theta)$ for Epochs $1-3$, only plotting $\theta$ values for polarizations above $0.08 \%$. Four features are present over the course of the three epochs, which we label Features $A, B, C$, and $D$. Feature $A$ is a strongly polarized feature in the 4550-4950 A wavelength range during Epoch 1, but it fades so that by Epoch 3 the feature has disappeared. The position angle for Feature $A$ is consistent with the emission shortward of $6000 \AA$. Feature $B$ is in the $5900-6250 \AA$ wavelength range, a feature normally associated with the familiar Si II $\lambda 6355 \AA$ absorption line. Feature $B$ strengthens so that by Epoch 3 it is the sole, dominant line feature. By Epoch 4, it has disappeared (Figure 1). During Epoch 1, the polarization angle for Feature $B$ changes from $\sim 70^{\circ}$ to $\sim 0^{\circ}$ on either side of this feature. Feature $C$ is narrower, in the 4200-4400 $\AA$ wavelength range, and evolves as if it is a weaker companion of Feature $A$. Feature $D$ is a broad but initially poorly defined feature in the 5000-5500 ^ wavelength range. The feature is most apparent during Epoch 2 and has disappeared by Epoch 3. Epoch 3 is notable both for the singular dominance of Feature $B$, but also because the polarization angle change between the blue and red halves of the spectrum that was seen in Epochs 1 and 2 has disappeared. We reiterate that Epoch 4 was not fitted with SYNAPPS as there are no line polarization features (Figure 3 ).

To understand the nature of these features, we compare them with the evolution of the individual spectral features, as explored with SYNAPPS. The SYNAPPS fitting supports that Feature $B$ is a $\mathrm{Si}$ II feature. We accept that association and label Feature $B$ as $P_{\mathrm{Si} \text { II }}$ throughout the remainder of this work. It is immediately clear that there are a few candidates to explain the polarization in Features $A, C$, and $D$.

Feature $A$ is located in a rapidly evolving portion of the spectrum where the absorption lines of S II, Fe II, and Si II overlap. HV Si II and photospheric Si II are present over the indicated wavelength range in Epoch 1, but by Epoch 2 the absorption from HV Si II is no longer evident. Perhaps this is why the polarization is not as well defined at the bluer wavelengths in Epoch 2 as the opacity, and therefore the covering factor, of HV Si II decreases. S II and HV Fe II show multiple absorption features over the wavelength range of Feature $A$ and could therefore be contenders. Photospheric Fe II also has some absorption over this region, but the emission feature at shorter wavelengths must be included to explain the entire polarization feature in at least Epoch 1.

By examining the individual flux spectra of Epoch 1, we see that $\mathrm{Mg}$ II and $\mathrm{HV}$ Fe II are the ions with absorption features most contained within the defined wavelength interval of Feature $C$. Photospheric Fe II and Fe III, however, also have absorption features that at least partially fall across the wavelength range. S II is the only ion with absorption features associated with Feature $D$. We note that photospheric and HV Fe II and Fe III have emission components across this range. 
Table 2

SYNAPPS Fit Parameters

\begin{tabular}{|c|c|c|c|c|c|}
\hline Ion & $\tau$ & $\begin{array}{c}v_{\min } \\
\left(10,000 \mathrm{~km} \mathrm{~s}^{-1}\right)\end{array}$ & $\begin{array}{c}v_{\max } \\
\left(10,000 \mathrm{~km} \mathrm{~s}^{-1}\right)\end{array}$ & $\begin{array}{c}v_{e} \\
\left(10,000 \mathrm{~km} \mathrm{~s}^{-1}\right)\end{array}$ & $\begin{array}{c}T \\
(1000 \mathrm{~K})\end{array}$ \\
\hline \multicolumn{6}{|c|}{ Epoch 1} \\
\hline Si II & 22.4 & 12.5 & 26.0 & 2.0 & 10 \\
\hline S II & 3.31 & 12.5 & 15.1 & 2.0 & 10 \\
\hline $\mathrm{Fe}$ II & 1.38 & 12.5 & 23.1 & 2.0 & 10 \\
\hline C II & 0.05 & 12.5 & 30.0 & 2.0 & 10 \\
\hline Mg II & 3.72 & 12.5 & 17.9 & 2.0 & 10 \\
\hline Si III & 3.09 & 12.5 & 15.0 & 2.0 & 10 \\
\hline $\mathrm{Fe}$ III & 2.88 & 12.5 & 25.5 & 2.0 & 10 \\
\hline HV Si II & 0.71 & 19.5 & 30 & 7.13 & 10 \\
\hline $\mathrm{HV}$ Fe II & 0.47 & 18.6 & 30 & 6.01 & 10 \\
\hline \multicolumn{6}{|c|}{ Epoch 2} \\
\hline Si II & 5.62 & 11.4 & 26.3 & 2 & 10 \\
\hline S II & 1.38 & 11.4 & 30 & 2 & 10 \\
\hline $\mathrm{Fe}$ II & 0.83 & 11.4 & 21 & 2 & 10 \\
\hline C II & 0.01 & 11.4 & 23.6 & 2 & 10 \\
\hline Mg II & 1.66 & 11.4 & 23.1 & 2 & 10 \\
\hline Si III & 2.0 & 11.4 & 15 & 2 & 15 \\
\hline $\mathrm{Fe}$ III & 1.12 & 11.4 & 18.8 & 2 & 15 \\
\hline HV Si II & 3.16 & 23.3 & 30 & 3.22 & 10 \\
\hline HV Fe II & 0.63 & 19.6 & 30.0 & 6 & 10 \\
\hline \multicolumn{6}{|c|}{ Epoch 3} \\
\hline $\mathrm{Si}$ II & 12.6 & 10.5 & 15 & 2 & 7 \\
\hline S II & 1.45 & 10.5 & 28 & 2 & 7 \\
\hline $\mathrm{Fe}$ II & 2 & 10.5 & 20 & 2 & 7 \\
\hline C II & $\ldots$ & $\cdots$ & $\cdots$ & $\cdots$ & $\cdots$ \\
\hline Mg II & 1.32 & 10.5 & 16.2 & 2 & 7 \\
\hline Si III & 0.83 & 10.5 & 15 & 2 & 10 \\
\hline Fe III & 0.74 & 10.5 & 15 & 2 & 10 \\
\hline $\mathrm{Na} \mathrm{I}$ & 0.47 & 10.5 & 26.9 & 2 & 7 \\
\hline
\end{tabular}

The elaborate blend of several elements in the flux spectrum of SN 2011fe does not allow us to determine with certainty if one particular element is the root of the ejecta asymmetrie$\mathrm{s}$; however, we explore three possibilities, which we label as Interpretations 1, 2, and 3. In Interpretation 1, we assign Feature A to Si II absorption from a complex of Si II absorption lines (rest frame $\lambda 5041 \AA, \lambda 5056 \AA, \lambda 5113 \AA$ ) seen in SYNAPPS fits. If photospheric Si II were the cause of line polarization at $4800 \AA$, then each absorption feature of this element at optical wavelengths can be connected to line polarization features in Epochs 1 and 2. This includes the small feature at $5700 \AA$ that has been associated with Si II $\lambda 5958$ and $\lambda 5979$ in SN 2004dt (Wang et al. 2006). However, to explain the broad polarization in Epoch 1 at $4800 \AA$, we must include HV Si II in Feature $A$. Unfortunately, the other absorption of this HV ion at $5900 \AA$ is not associated with line polarization, which is a point of conflict for this interpretation. Similarly, if Si II explains both Features $A$ and $B$, then it is hard to explain the changing difference in angle between these two components. Si II is also not a viable candidate to explain Feature $C$ or $D$. For Interpretation 1 , we speculate that Feature $A$ traces the same asymmetric layers in the SN atmosphere that are seen in Feature $B$ in Epoch 2 and 3, but at an earlier time. This may be an important clue to the changes revealed by the receding SN photosphere, which may be able to provide constraints on radiative transfer models of the observed polarization behavior.
Interpretation 2 assigns Feature $A$ to Fe II and Fe III. We note that the shifting absorption features of the iron complexes could explain why Feature $A$ is not as well defined in Epoch 2 as compared to Epoch 1. Feature $C$ is then also explained by Fe II and Fe III, with the relative strengths between Features $A$ and $C$ matching the absorption rather well. HV Fe II is also a potential contributor, although the second absorption from HV Fe II of the SYNAPPS fits shifts to the blue and therefore causes part of the feature to move beyond the peak of the polarization feature. A similar situation occurs for photospheric Fe II and Fe III, but the effect is to shift the absorption profiles into better sync with the polarization feature. In general, the iron complexes absorb over a broad wavelength range and therefore make it difficult to match line polarization features with individual absorption features.

Interpretation 3 assigns Feature $A$ to $\mathrm{S}$ II. There is strong absorption of S II where the line polarization feature appears, but if $\mathrm{S} I \mathrm{II}$ is also to explain Feature $D$, there is the issue that the polarization strength ratio does not match the ratio of the strength of the two broad absorption components.

The interpretations we outline here have been mentioned in previously published studies of spectropolarimetry of other $\mathrm{SNe}$ Ia (see Section 4). There is not enough information to confidently distinguish between the three interpretations for SN 2011fe, but we are hopeful that this data set can be studied in combination with other normal $\mathrm{SNe}$ Ia that we have 


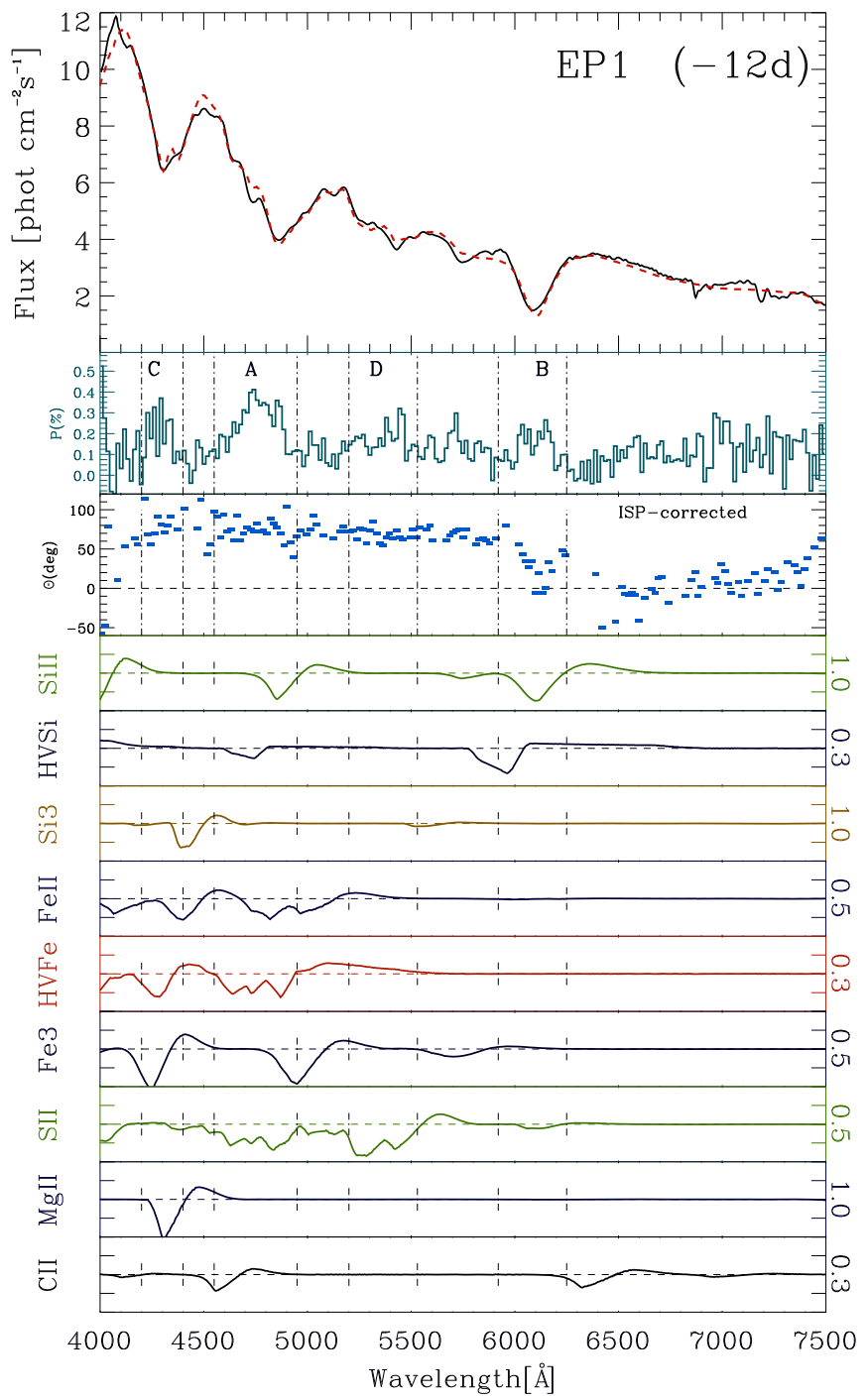

Figure 4. Upper panel: flux spectrum of Epoch 1 fit with the SYNAPPS algorithm. The flux spectrum is shown with a black solid line, and the SYNAPPS fit is shown as a red dashed line. Fit parameters are listed in Table 2. Lower panels: polarization and $\theta$ spectra compared against individual ions from SYNAPPS fits for Epoch 1. The ion flux values are plotted on different scales, as shown on the right-hand axis. The ISP-corrected $P$ and $\theta$ spectra have been binned into $16 \AA$ bins. See text for explanation of $A, B, C$, and $D$ labels.

observed to determine if any of the three possibilities are favored in general.

Regardless of the interpretion of the source of the features, the line polarization can be further studied by plotting the evolution of Features $A$ and $B$ in $q-u$ space (Figure 7). In Epochs 2 and 3, the $\lambda 6355 \AA$ line is seen to exhibit a loop in the polarization (top panel). Interestingly, the loop appears counterclockwise when moving blue to red during Epoch 2, but the same loop rotates clockwise in Epoch 3. As loops are suggested to be present when there is a deviation from axial symmetry, the Si II distribution appears to deviate from spherical symmetry, but without exhibiting dominant axial symmetry. The lower panel shows the same presentation for Feature $A$, in this case exhibiting a hint of multiple loops during Epoch 1 in the same quadrant as Feature $A$. Since in all of the interpretations Feature $A$ is actually a complex of lines, it is not clear that a clean loop should be expected.

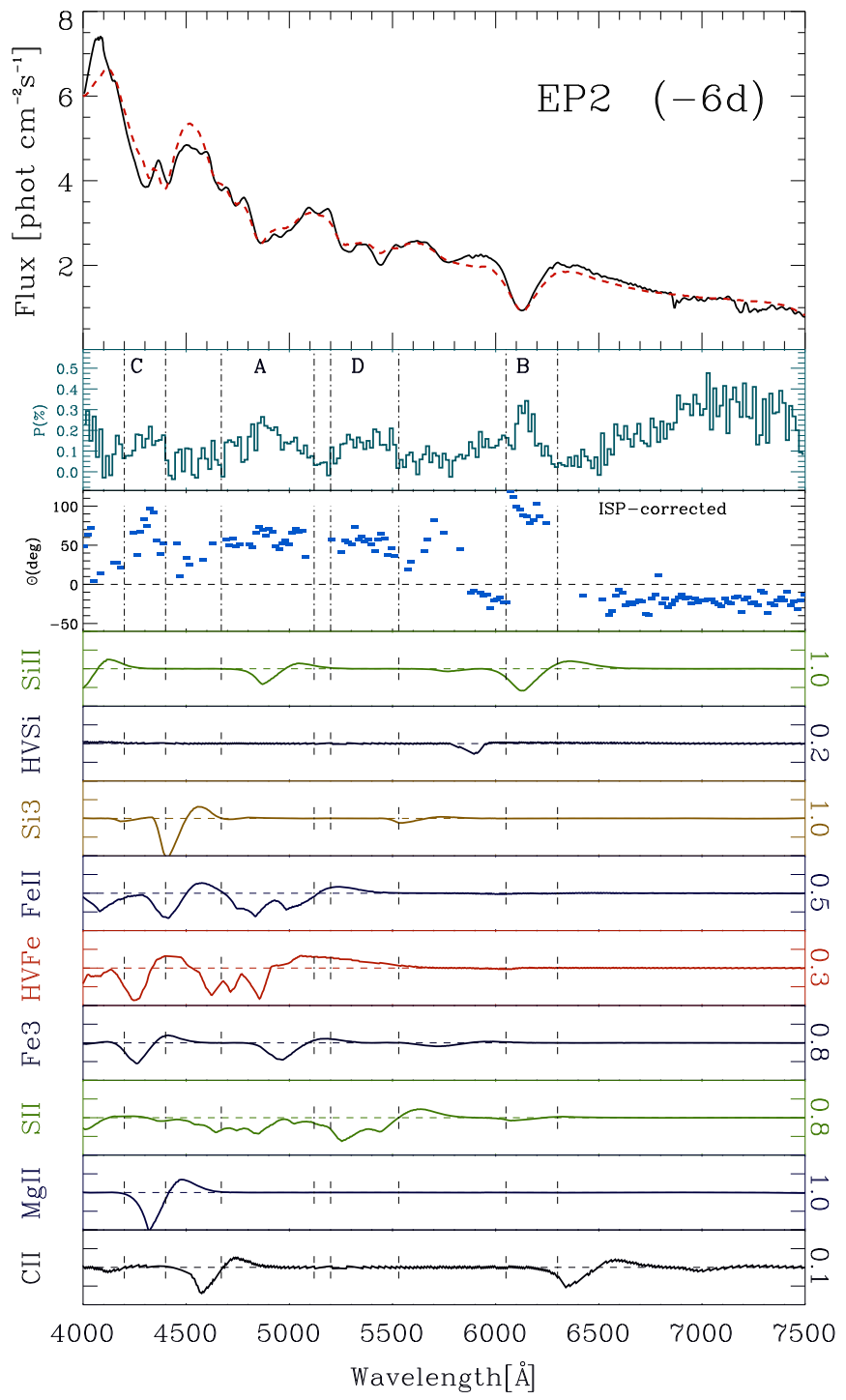

Figure 5. Upper panel: flux spectrum of Epoch 2 fit with the SYNAPPS algorithm. The flux spectrum is shown with a black solid line, and the SYNAPPS fit is shown as a red dashed line. Fit parameters are listed in Table 2. Lower panels: polarization and $\theta$ spectra compared against individual ions from SYNAPPS fits for Epoch 2. The ion flux values are plotted to different scales, as shown on the right-hand axis. See text for explanation of $A, B, C$, and $D$ labels.

\subsection{Evolution of Continuum Polarization}

The red wavelengths of the continuum emission exhibit polarization reaching up to $\sim 0.4 \%$ in Epoch 2 . The wealth of line polarization features in $\mathrm{SN} 2011 \mathrm{fe}$ makes it difficult to measure the continuum polarization at shorter wavelengths, but there is the suggestion of polarization at the $\sim 0.1 \%$ level between Features $C$ an $A$ in Epochs 1-3 (Figures 4-6). A similar level of red continuum emission was reported for SNe 1999by (Howell et al. 2001), 2001el (Wang et al. 2003), 2004S (Chornock \& Filippenko 2008), and 2005ke (Patat et al. 2012). In Figure 8, we show red-continuum polarization of SN 2011fe during Epoch 2 compared to SNe 1999by and $2005 \mathrm{ke}$, with $2011 \mathrm{fe}$ providing a reasonable match with $2005 \mathrm{ke}$. The increase in polarization toward longer wavelengths has been proposed as being due to an overall oblate geometry combined with the decreased importance at red wavelengths of line scattering depolarizing the observed light 


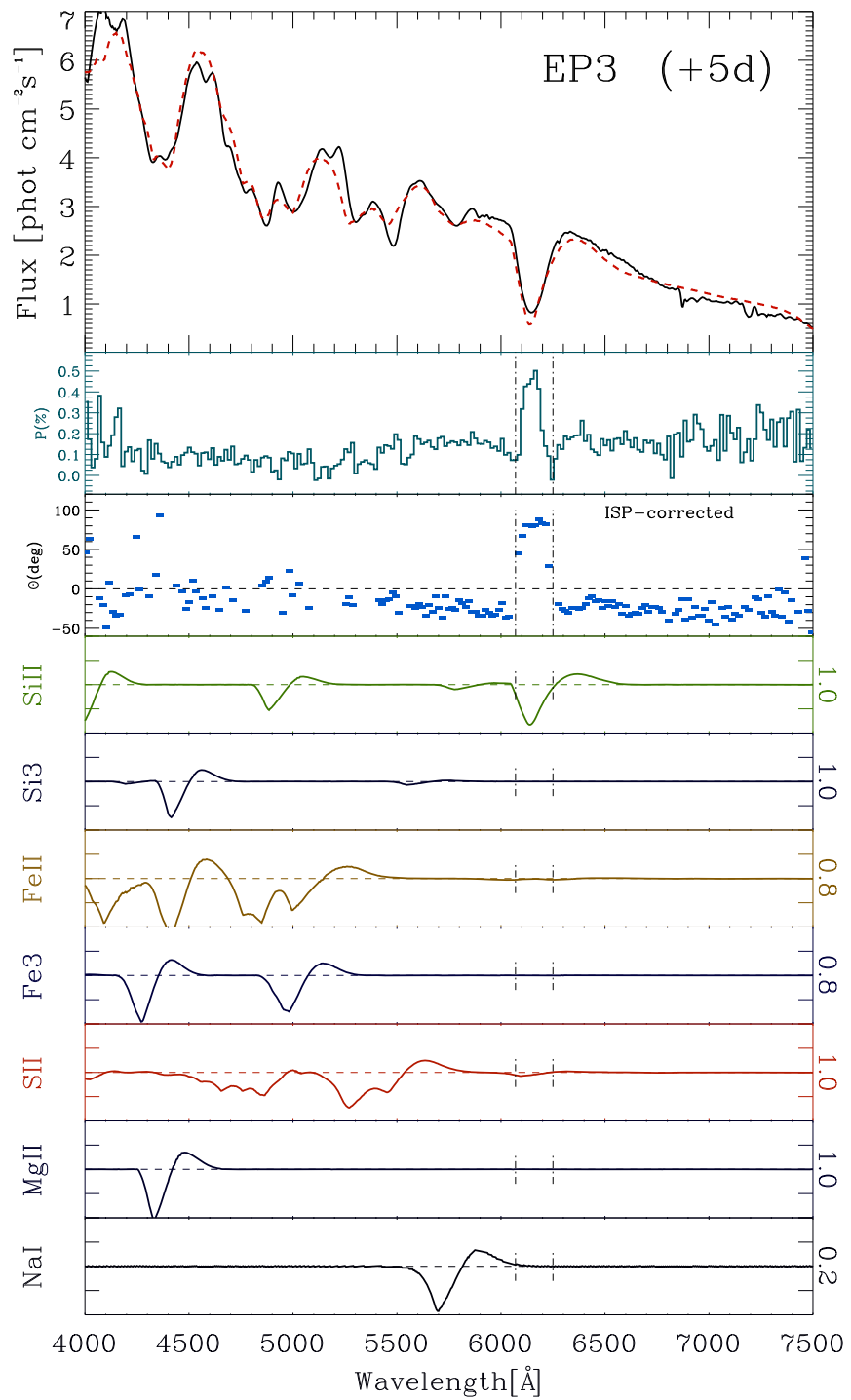

Figure 6. Upper panel: flux spectrum of Epoch 3 fit with SYNAPPS algorithm. The flux spectrum is shown with a black solid line, and the SYNAPPS fit is shown as a red dashed line. Fit parameters are listed in Table 2. Lower panels: polarization and $\theta$ spectra compared against individual ions from SYNAPPS fits for Epoch 3. The ion flux values are plotted to different scales, as shown on the right-hand axis. See text for explanation of $A, B, C$, and $D$ labels.

(Wang et al. 1997). This hypothesis has been theoretically supported (e.g., Patat et al. 2012; Bulla et al. 2015) and suggests that longer wavelengths are the best for studying the overall degree of asphericity in the $\mathrm{SN}$ ejecta. The continuum polarization in the $6300-7400 \AA$ wavelength range varied with epoch, perhaps best presented in the form of a $q-u$ scatter plot (Figure 9). The continuum polarization is evident during Epochs 1 and 2, but is absent by Epoch 4. Linear fits to the Epochs 1 and 2 data suggest a change in angle of $\sim 20^{\circ}$, although the linearity of the scatter is only apparent in Epoch 2 . The Bulla et al. (2015) oblate geometry model features the polarization of the red continuum dropping between 15 days postexplosion to 25 days postexplosion. The time variability of the continuum polarization seen in SN 2011fe might be evidence of an oblate geometry. It would be interesting to determine the level of oblateness that best describes SN 2011fe and then revisit the Patat et al. (2013) study of the NaD variability, but with an oblate rather than spherical emitting region.
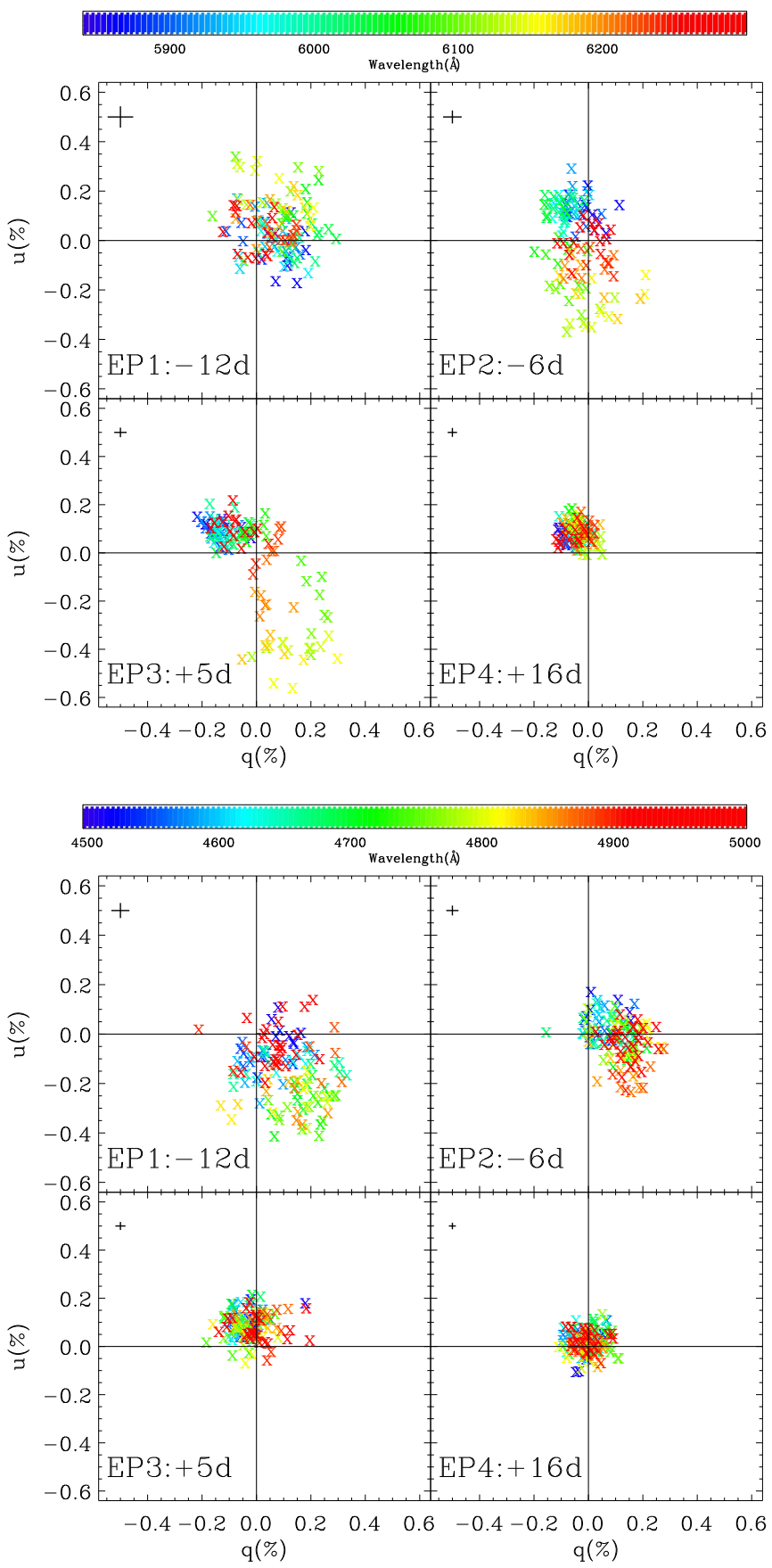

Figure 7. Polarization features $B$ and $A$ plotted in the $q-u$ plane. The upper panel shows the $\lambda 6355 \AA$ line (Feature $B$ ) for all four epochs, plotted over 5840-6300 $\AA$. The lower panel shows the feature $A$ for all four epochs, plotted over $4500-5000 \AA$. The $\lambda 6355 \AA$ line exhibits clear loops during the second and third epochs, while there is a suggestion of multiple loops during Epoch 1 for Feature $A$. The average error bar across each line is shown in the upper left of each panel.

\section{Comparisons with Polarization in other SNe Ia}

\subsection{Line Features $A-D$}

The earliest line polarization feature, $A$, is most polarized during Epoch 1 and is gone by the third epoch. SN 2012fr, 2002bo, and SN 2006X are the only other normal SN Ia with published observations at similar epochs. Maund et al. (2013) report no line features in that wavelength range for 




Figure 8. Red continuum polarization of SN 2011fe (-6d) compared to SN 1999by (0d) and SN 2005ke (-8/-7d). SN 2011fe continuum polarization (solid blue line) roughly matches SN 2005ke (dot-dashed red line; Patat et al. 2012) and shows a similar increase with wavelength as SN 1999by (dashed green line; Howell et al. 2001). Errors of the SN 2011fe data are shown in the lower panel.



Figure 9. The red continuum, 6300-7400 $\AA$, in the $q-u$ plane for Epochs 1-4. There is an indication of axial symmetry in the Epoch 2 polarization, pointing in a different direction than seen in Epoch 1. Dashed lines show linear fits to the data points. The Epoch 4 data are not aligned with the origin, likely due to using a constant rather than an actual Serkowski function when performing ISP subtraction.

SN 2012fr; indeed they use the 5100-5300 wavelength range as an "intrinsically depolarized" region. Similarly, no equivalent line features are apparent for SN 2002bo in Figure 1 of Wang et al. (2007). A daily sequence of VLT spectropolarimetry of SN 2006X does show a polarized feature in that wavelength range (referred to as $\lambda 5051 \AA$ in that work), with the polarization reaching $0.37 \%$ at -6 days. SN 2006X's feature was narrower and did not show a well-defined peak as in SN 2011fe. SNe 2002bo and 2006X are of the HVG/HV group, so comparisons with $2011 \mathrm{fe}$ are perhaps less appropriate than comparisons with SN 2012fr, a member of the LVG/LV group, as the $\mathrm{HV} / \mathrm{LV}$ differentiation is based on the prepeak

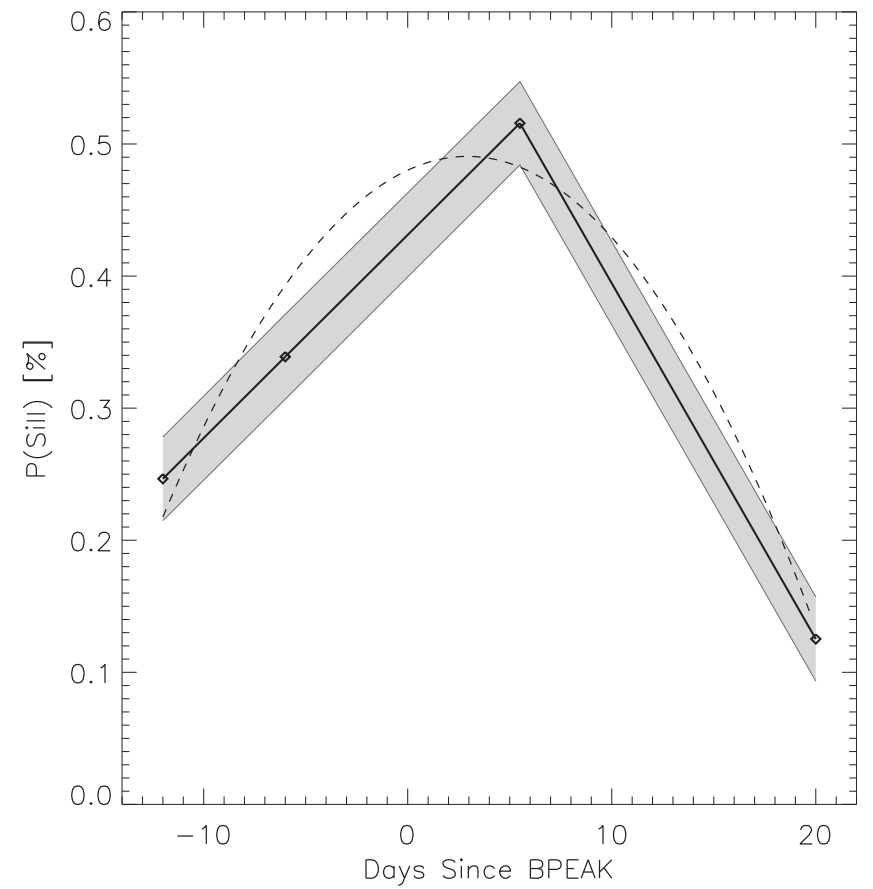

Figure 10. The time evolution of the polarization of the Si II $\lambda 6355 \AA$ absorption feature. Whereas some SNe Ia studied in Wang et al. (2007) were found to reach the largest $\mathrm{Si}$ II polarization roughly 5 days before the optical maximum, SN 2011fe clearly reaches the largest polarization after the optical maximum, if assuming a parabolic fit to the data with a shape similar to Wang et al. (2007; dashed line). Error bars were generated by determining the standard deviation of $P_{\mathrm{Si} \text { II }}$ estimations when $q(\mathrm{ISP})$ and $u($ ISP) change according to the averages from Epochs 4-9, as shown in Figure 2. A continuum polarization level of $\sim 0.08 \%$ was not subtracted from $P_{\mathrm{Si} \mathrm{II}}$.

epochs. However, the presence or absence of Feature $A$ in early-epoch spectropolarimetry does not appear to be strictly correlated with the $\mathrm{HV} / \mathrm{LV}$ categorization. It is worth mentioning that SN 2012fr is a NUV-red event without a detection of unburned carbon (see Brown et al. 2014 [SOUSA] for UVOT photometry and Childress et al. 2013 for a spectral study), whereas SN 2011fe is a NUV-blue event with a detection of unburned carbon (Milne et al. 2013; Pereira et al. 2013). A handful of supernovae have also shown line polarization near $4800 \AA$, but at postmaximum epochs $(+9$ days and later for SNe 1997dt, 2003du, and 2004S), causing the feature to be identified as Fe II (Leonard et al. 2005). Each of these supernovae only had a single epoch of observation, so we cannot say if the line polarization was observable at earlier epochs.

Historically, the peak of the Si II $\lambda 6355 \AA$ line, Feature $B$, has been used as the measure of $P_{\mathrm{Si} \text { II }}$. Although this introduces a dependence on spectral resolution that would be improved through the use of equivalent widths, for the sake of comparison with other SNe Ia in the literature, in this work we also use this method. As shown in Figure 10, the evolution of the peak polarization of the $\lambda 6355 \AA$ line is $0.24 \%, 0.34 \%$, and $0.52 \%$ for Epochs $1-3$, respectively. In that figure, we generate error bars by determining $P_{\mathrm{Si} \text { II }}$ for each point in a grid of estimates when $q$ (ISP) and $u$ (ISP) are each varied by $\pm 0.05 \%$, reflecting the variation of the $q$ (ISP) and $u$ (ISP) estimates shown in Figure 2. The error bars are then the standard deviation of the estimates in that grid, and at a level of $\sim 0.03 \%$, they show that the ISP estimation does not appreciably affect the evolution of $P_{\mathrm{Si} \text { II }}$. We keep with the 
formalism of $P_{\mathrm{Si} \text { II }}$ estimation in the literature and do not subtract the continuum polarization (which we estimate to be $\sim 0.08 \%$ ). Thus, by Epoch 4, the polarization is consistent with zero. This evolution is surprising, as earlier works presented $\mathrm{SNe}$ Ia for which Feature $B$ clearly peaks before the optical maximum (Wang et al. 2007 Patat et al. 2009). However, our time sequence clearly favors this feature reaching a maximum after the optical peak, which is fundamentally different than the scenario presented in those previous works. Porter et al. (2016) reported that $\mathrm{SN} 2014 \mathrm{~J}$ also features a late-peaking $P_{\mathrm{Si} \text { II }}$, so we do not consider SN 2011fe to be anomalous.

Concentrating on Feature $C, \mathrm{Mg}$ II $\lambda 4471 \AA$ was polarized in SN 2006X, where the line polarization extended over a narrower range than SN 2011fe's Feature $C$ between 4150 and $4300 \AA$ (Wang et al. 2007). The authors mention the feature is significant only at -3 and -1 days when it peaked at $0.5 \%$, but was present starting as early as -8 days. Mg II was also polarized in SN 2004dt at -7 days between 4100 and $4300 \AA$ and reached a peak of $\sim 1 \%$ polarized (Wang et al. 2006).

Feature $D$ is mentioned as being due to $\mathrm{S}$ II for $\mathrm{SNe} 2005 \mathrm{ke}$ (Patat et al. 2012) and 2012fr (Maund et al. 2013), but not in SN 2004S (Chornock \& Filippenko 2008).

In terms of the relative evolution of the features, for SN 2011fe, Feature $B$ increases in polarization as Feature $A$ decreases, whereas in SN 2006X, Feature $A$ is always weaker than Feature $B$.

To summarize, although most of the attention of $\mathrm{SNe}$ Ia polarization literature has focused on the Si II $\lambda 6355 \AA$ and $\mathrm{Ca}$ II lines, Features $A, C$, and $D$ as identified in SN 2011fe are present in other fairly well observed SNe Ia with polarization measurements. Specifically, Mg II has been correlated with Feature $C, \mathrm{~S}$ II with Feature $D$, and Feature $A$ has been matched with $\mathrm{Si}$ II at premaximum epochs and Fe II postmaximum.

\subsection{Previously Reported Correlations}

SN $2011 \mathrm{fe}$ can be included with other SNe Ia in studies of the time evolution of Si II $\lambda 6355 \AA$ polarization. Wang et al. (2007) reported a postive correlation between the width of the optical peak and the strength of the Si II $\lambda 6355 \AA$ line polarization at -5 days $\left(P_{\mathrm{Si}}\right)$. For $\mathrm{SN} 2011 \mathrm{fe}$, Munari et al. (2013) reported $\Delta m_{15}(B)=1.108$, Richmond \& Smith (2012) reported $\Delta m_{15}(B)=1.2$, and Pereira et al. (2013) reported $\Delta m_{15}(B)=1.103$. Using a rough average of those values, $\Delta m_{15}(B)=1.15$, the Wang et al. (2007) formula predicts $P_{\text {Si II }}=0.55 \%$. This is consistent with our maximum measured polarization, $0.52 \%$ at +5 days. Since Wang et al. (2007) chose -5 days as their standard epoch, and we found SN $2011 \mathrm{fe}$ peaks after the optical peak, we will also consider equally the -6 day polarization, which we measure to be $0.34 \%$. Because the temporal sampling of Si II $\lambda 6355 \AA$ polarization is so poor for most $\mathrm{SNe}$ Ia, for comparisons with other $\mathrm{SNe}$, we consider SN 2011 fe to be between $0.34 \%$ and $0.52 \%$, the -5 day and highest measured values, respectively. In the upper left panel of Figure 12, we show these two choices of $P_{\mathrm{Si} \text { II }}$ plotted versus $\Delta m_{15}(B)$ with other SNe Ia, concluding that SN $2011 \mathrm{fe}$ falls within the scatter of the relation for either choice of $P_{\mathrm{Si} \text { II }}$.

SN 2011fe also falls within the scatter of other SNe Ia for $P_{\text {Si II }}$ plotted versus the equivalent width of Si II $\lambda 6355 \AA$. That relation tests the strength of polarization as being only due to the line strength. In the lower left panel of Figure 12, we treat the Maund et al. (2010) suggestion of a linear correlation

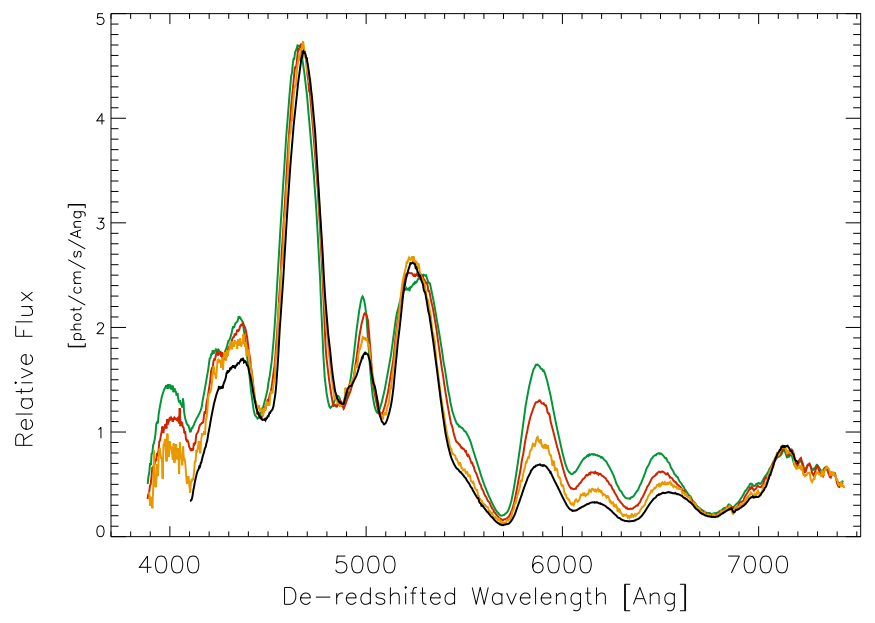

Figure 11. Spectral sequence of the nebular flux spectra of SN $2011 \mathrm{fe}$. Epochs 7-10 show the evolution of forbidden [Fe] and [Co] lines. Epochs 7-10, colored as 7 (green), 8 (red), 9 (orange), and 10 (black), are normalized to the $\sim 4600 \AA$ line. The progression of the [Co III] complex in the 5700-6700 ^ wavelength range is apparent.

between the Si II velocity gradient (e.g., $\dot{v}_{\mathrm{Si} \text { II }}$ : HVG/LVG) and $P_{\mathrm{Si} \mathrm{II}}$. Pereira et al. (2013) reported $\dot{v}_{\mathrm{Si} \mathrm{II}}=59.6 \pm$ $3.2 \mathrm{~km} \mathrm{~s}^{-1} \mathrm{~d}^{-1}$, leading to a predicted $P_{\mathrm{Si} \text { II }}=0.62 \%$. This is again slightly larger than our measured value of $0.52 \%$, as seen in the lower left panel of Figure 12, but SN 2011fe falls comfortably within the scatter of the other SNe Ia plotted.

Maund et al. (2010) also investigated a linear correlation between the strength of the -5 day $\mathrm{Si}$ II $\lambda 6355 \AA$ line polarization and the nebular phase velocity of Fe-group elements, based upon a claimed correlation between the nebular velocity and the velocity gradient of the Si II $\lambda 6355 \AA$ absorption feature by Maeda et al. (2010). Maund et al. (2010) specifically measured the shifting of the [Fe II] $\lambda 7155 \AA$ and [Ni II] $\lambda 7378 \AA$ emission lines. The wavelength range of our spectra (Figure 11) only permits the study of the [Fe II] $\lambda 7155 \AA$ line; we find shifts of $-1128,-1149$, and $-1601 \mathrm{~km} \mathrm{~s}^{-1}$ for Epochs 5, 6, and 7, respectively. The lower panel of Figure 12 shows that SN 2011fe is similar to a number of other SNe Ia that have a measurement of the [Fe II] $\lambda 7155 \AA$ shift, but as noted by Maund et al. (2010), there is no clear correlation between these parameters. SNe 2001V and 2004dt are identified because each is peculiar, and they are not considered members of the "normal" group.

Despite peaking at a later epoch than the Wang et al. (2007) standard epoch, the Si II $\lambda 6355 \AA$ polarization of SN $2011 \mathrm{fe}$ falls to zero by +16 days. Spectra of normal SNe Ia at a similar epoch were presented for $\mathrm{SNe} 1997 \mathrm{dt}(+21 \mathrm{~d})$ and $2003 \mathrm{du}$ $(+18 d)$ by Leonard et al. (2005), for SN 2002 bo $(+14 d)$ by Wang et al. (2007), and for SN 2012fr (+24d) by Maund et al. (2013). These comparison spectra all show some evidence for Si II $\lambda 6355 \AA$ polarization, although for SN 2012fr the feature is quite weak. Porter et al. (2016) further investigated the time evolution of the Si II $\lambda 6355 \AA$ polarization for a collection of $\mathrm{SNe}$ Ia.

\section{Summary}

By virtue of being a very nearby SN Ia that was discovered at an extremely early epoch, SN $2011 \mathrm{fe}$ has proven to be one of the best-studied SNe Ia of all time. Spectropolarimetry of this 



Figure 12. Polarization of the Si II $\lambda 6355 \AA$ absorption feature compared with potentially correlated parameters. Wang et al. (2007) claimed a correlation between $P_{\mathrm{Si} \text { II }}$ and peak width (upper left panel). It has been suggested that there is a correlation between $P_{\mathrm{Si} \text { II }}$ and the equivalent width of the Si II $\lambda 6355 \AA$ absorption feature (upper right panel). Maund et al. (2010) claimed a correlation between $P_{\mathrm{Si} \text { II }}$ and the velocity gradient of the Si II $\lambda 6355 \AA$ absorption feature (lower left panel). Maund et al. (2010) also investigated claims of a correlation between $P_{\mathrm{Si}}$ and the nebular velocity of the emission feature, [Fe II] $7155 \AA$ A (lower right panel). The filled boxes show our measured values for SN $2011 \mathrm{fe}$. The range in polarization reflects the difference between the -5 day polarization and the maximum measured polarization. The range in peak width and $\dot{v}_{\mathrm{Si}}$ are from the literature. The range in $v_{\mathrm{NEB}}$ is from the minimum and maximum measured in our Epoch 5-7 nebular spectra. Non2011fe data are from Wang et al. (2007), Pereira et al. (2013), Silverman et al. (2012), Hachinger et al. (2006), and Maund et al. (2010) and references therein.

SN obtained over four epochs has revealed both line and continuum polarization, with both components exhibiting time variability.

Utilizing the SYNAPPS algorithm, we present three possible interpretations for the line polarization features in the spectra from the initial epoch to later epochs with no line polarization. Interestingly, the maximum polarization of the common Si II $\lambda 6355 \AA$ feature $(B)$ happens at a later epoch than has been seen for other SNe Ia.

These polarization features add to the study of this wellobserved, normal, NUV-blue, LV, unburned-carbon-bearing SN Ia. Over the course of a multiyear campaign, we plan to observe enough $\mathrm{SNe}$ Ia to search for similarities and differences between individual events as a function of known parameters, exploring which characteristics of an SN Ia explosion drive polarization.

This work was partially supported by NSF Collaborative Research Award \#AST-1210599. PAM acknowledges support from NASA ADP grant NNX10AD58G. PSS acknowledges support from NASA/Fermi Guest Investigator grant NNX09AU10G. NS received partial support from NSF grant AST-1515559. B.T.J. acknowledges support from the NSF, through its funding of NOAO, which is operated by AURA, Inc. under a cooperative agreement with the NSF. Some observations reported here were obtained at the MMT
Observatory, a joint facility of the University of Arizona and the Smithsonian Institution.

\section{References}

Benetti, S., Cappellaro, E., Mazzali, P. A., et al. 2005, ApJ, 623, 1011 Bloom, J., Kasen, D., Shen, K. J., et al. 2012, ApJ, 744, 17

Branch, D., Baron, E., Hall, N., Melakayil, M., \& Parrent, J. 2005, PASP, 117,545

Branch, D., Fisher, A., \& Nugent, P. 1993, AJ, 106, 2383

Brown, P. J., Breeveld, A. A., Holland, S., Kuin, P., \& Pritchard, T. 2014, Ap\&SS, 354, 89

Brown, P. J., Dawson, K. S., de Pasquale, M., et al. 2012a, ApJ, 749, 22

Brown, P. J., Roming, P. W. A., Milne, P. A., et al. 2012b, ApJ, 753, 22

Bulla, M., SIm, S. A., \& Kromer, M. 2015, MNRAS, 450, 967

Cenko, S. B., Thomas, R. C., Nugent, P. E., et al. 2011, ATel, 3583, 1

Childress, M. J., Aldering, G., Antilogus, P., et al. 2013, ApJ, 770, 29

Chornock, R., \& Filippenko, A. V. 2008, AJ, 136, 2227

Cox, N. L. J, \& Patat, F. 2014, A\&A, 565, 61

Folatelli, G., Phillips, M. M., Morrell, N., et al. 2012, ApJ, 745, 74

Foley, R. J., \& Kasen, D. 2011, ApJ, 729, 55

Foley, R. J., \& Kirshner, R. P. 2013, ApJL, 769, L1

Hachinger, S., Mazzali, P., \& Benetti, S. 2006, MNRAS, 370, 299

Howell, A., Höflich, P., Wang, L., \& Wheeler, J. C. 2001, ApJ, 556, 302

Iwamoto, K., Brachwitz, F., Nomoto, K., et al. 1999, ApJS, 125, 439

Leonard, D. C., Li, W., Filippenko, A. V., Foley, R. J., \& Chornock, R. 2005, ApJ, 632, 450

Li, W., Bloom, J. S., Podsiadlowski, P., et al. 2011, Natur, 480, 348

Livio, M., \& Pringle, J. E. 2011, ApJ, 740, 18

Maeda, K., Benetti, S., Stritzinger, M., et al. 2010, Natur, 466, 82

Matheson, T., Joyce, R. R., Allen, L. E., et al. 2012, ApJ, 754, 19 
Maund, J. R., Fraser, M., Smartt, S. J., et al. 2013, MNRAS, 433, L20

Maund, J. R., Höflich, P., Patat, F., et al. 2010, ApJ, 722, 1162

Milne, P. A., Brown, P. J., Roming, P. W. A., et al. 2013, ApJ, 779, 23

Munari, U., Henden, A., Belligoli, R., et al. 2013, NewAR, 20, 30

Nugent, P., Sullivan, M., Bersier, D., et al. 2011a, ATel, 3581, 1

Nugent, P., Sullivan, M., Cenko, S. B., et al. 2011b, Natur, 480, 344

Parrent, J. T., Howell, D. A., Friesen, B., et al. 2012, ApJ, 752, 26

Patat, F., Baade, D., Höflich, P., et al. 2012, A\&A, 545, 7

Patat, F., Chandra, P., Chevalier, R., et al. 2007, Sci, 317, 924

Patat, F., Cordiner, M. A., Cox, N. L. J., et al. 2013, A\&A, 549, A62

Patat, F., Höflich, P., Baade, D., et al. 2009, A\&A, 508, 229

Patat, F., Taubenberger, S., Cox, N. L. J., et al. 2015, A\&A, 577, 53

Pereira, R., Thomas, R. C., Aldering, G., et al. 2013, A\&A, 554, 27

Perlmutter, S., Aldering, G., Goldhaber, G., et al. 1999, ApJ, 517, 565

Phillips, M. M. 1993, ApJL, 413, L105

Porter, A. L., Leising, M. D., Williams, G., et al. 2016, ApJ, 828, 24
Richmond, M. W., \& Smith, H. A. 2012, JAVSO, 40, 872

Riess, A. G., Filippenko, A. V., Challis, P., et al. 1998, AJ, 116, 1009

Schmidt, G. D., Elston, R., \& Lupie, O. L. 1992a, AJ, 104, 1563

Schmidt, G. D., Stockman, H. S., \& Smith, P. S. 1992b, ApJL, 398, L57

Serkowski, K., Mathewson, D. S., \& Ford, V. L. 1975, ApJ, 196, 261

Silverman, J. M., Kong, J. J., \& Filippenko, A. V. 2012, MNRAS, 425, 1819

Simon, J. D., Gal-Yam, A., Gnat, O., et al. 2009, ApJ, 702, 1157

Tanaka, M., Kawabata, K. S., Yamanaka, M., et al. 2010, ApJ, 714, 1209

Thomas, R. C., Aldering, G., Antilogus, P., et al. 2011, ApJ, 743, 27

Wang, L., Baade, D., \& Patat, F. 2007, Sci, 315, 212

Wang, L., Baade, D., Höflich, P., et al. 2003, ApJ, 591, 1110

Wang, L., Baade, D., Höflich, P., et al. 2006, ApJ, 653, 490

Wang, X., Filippenko, A. V., Ganeshalingam, M., et al. 2009, ApJL, 699, L139

Wang, X., \& Wheeler, J. C. 2008, ARAA, 46, 433

Wang, L., Wheeler, J. C., \& Hoflich, P. 1997, ApJ, 476, 27

Wardle, J. F. C., \& Kronberg, P. P. 1974, ApJ, 194, 249 